\title{
Increased Smooth Muscle Kv11.1 Channel Check for updates Expression in Pulmonary Hypertension and Protective Role of Kv11.1 Channel Blocker Dofetilide
}

\author{
Nataliia V. Shults, Vladyslava Rybka, Yuichiro J. Suzuki, and Tinatin I. Brelidze
}

From the Department of Pharmacology and Physiology, Georgetown University Medical Center, Washington, District of Columbia

\author{
Accepted for publication \\ September 17, 2019. \\ Address correspondence to \\ Tinatin I. Brelidze, Ph.D., \\ Department of Pharmacology \\ and Physiology, Georgetown \\ University Medical Center, \\ 3900 Reservoir Rd NW, Med- \\ Dent Bldg., SE406, Washing- \\ ton, DC 20057. E-mail: tib5@ \\ georgetown.edu.
}

\begin{abstract}
Kv11.1 potassium channels are essential for heart repolarization. Prescription medication that blocks Kv11.1 channels lengthens the ventricular action potential and causes cardiac arrhythmias. Surprisingly little is known about the Kv11.1 channel expression and function in the lung tissue. Here we report that Kv11.1 channels were abundantly expressed in the large pulmonary arteries (PAs) of healthy lung tissues from humans and rats. Kv11.1 channel expression was increased in the lungs of humans affected by chronic obstructive pulmonary disease-associated pulmonary hypertension and in the lungs of rats with pulmonary arterial hypertension (PAH). In healthy lung tissues from humans and rats, Kv11.1 channels were confined to the large PAs. In humans with chronic obstructive pulmonary diseaseassociated pulmonary hypertension and in rats with PAH, Kv11.1 channels were expressed in both the large and small PAs. The increase in Kv11.1 channel expression closely followed the time-course of the development of pulmonary vascular remodeling in PAH rats. Treatment of PAH rats with dofetilide, an Kv11.1 channel blocker approved by the US Food and Drug Administration for use in the treatment of arrythmia, inhibited PAH-associated pulmonary vascular remodeling. Taken together, the findings from this study uncovered a novel role of Kv11.1 channels in lung function and their potential as new drug targets in the treatment of pulmonary hypertension. The protective effect of dofetilide raises the possibility of repurposing this antiarrhythmic drug for the treatment of patients with pulmonary hypertension. (Am J Pathol 2020, 190: 48-56; https://doi.org/10.1016/j.ajpath.2019.09.010)
\end{abstract}

Kv11.1 potassium selective channels, also known as EAGrelated gene channels, are best known for their function in the heart, where they are involved in the repolarization of the cardiac action potential. ${ }^{1-4}$ Inhibition of Kv11.1 currents, due to the genetically occurring mutations or as a side effect of a prescription medication, lengthens the QT interval on electrocardiogram, causing long QT syndrome, a potentially fatal cardiac arrhythmia. ${ }^{5-8} \mathrm{Kv} 11.1$ channels also play a role in cancer, in which inhibition of Kv11.1 channel expression and/or Kv11.1 currents decreases the proliferation of cancer cells. ${ }^{9,10}$ In addition to the cardiac tissue, Kv11.1 channels are also expressed in the brain, ${ }^{11,12}$ retina, ${ }^{13}$ gallbladder, ${ }^{14}$ stomach, ${ }^{15}$ and intestines. ${ }^{16-18}$ However, surprisingly little is known about Kv11.1 channel expression and function in normal lung tissue.
Potassium channels are essential for proper lung function, and defects in ion channels have been linked to various respiratory diseases. ${ }^{19}$ Potassium channels are especially intimately involved in vascular remodeling in pulmonary hypertension, including pulmonary arterial hypertension (PAH) and chronic obstructive pulmonary disease (COPD)associated hypertension. ${ }^{20-22}$ PAH and COPD are fatal diseases associated with narrowing of the arteries in the lung, with no known cure. ${ }^{23-25}$ Several types of potassium

\footnotetext{
Supported by National Institute of General Medicine grant R01GM124020 (T.I.B.) and National Heart, Lung, and Blood Institute grant R01HL072844 (Y.J.S.).

Disclosures: All authors have a pending patent application on the use of dofetilide for pulmonary hypertension treatment (U.S. Patent Application No. $62 / 852,020)$.
} 
channels have been implicated in vascular remodeling in $\mathrm{PAH}$ and COPD, including big potassium (BK) channels, $\mathrm{K}_{\text {ATP }}$ channels, and two-pore domain potassium channels. ${ }^{26}$ The decrease in currents through potassium channels in response to hypoxia leads to the cell depolarization that increases $\mathrm{Ca}^{2+}$ influx through the voltage-gated calcium channels, causing vasoconstriction. ${ }^{26,27}$

Despite the importance of Kv11.1 channels for many physiologic processes, their expression and function in the pulmonary vasculature and their potential role in PAH- and COPD-associated vascular remodeling have not been investigated. Here we report that Kv11.1 channels were expressed in the lung vasculature of healthy humans and rats. Kv11.1 expression was detected in large pulmonary arteries (PAs; diameter $\geq 100 \mu \mathrm{m}$ ), and was undetectable in smaller PAs (diameter $<100 \mu \mathrm{m}$ ), in both healthy humans and rats. In humans with COPD-associated pulmonary hypertension and in rats with PAH, Kv11.1 channel expression levels were increased and were detected not only in the large-diameter PAs but also in the smaller PAs. The increases in Kv11.1 channel expression levels closely followed the time-course of vascular remodeling in PAH rats. Surprisingly, treatment of PAH rats with the Kv11.1 channel-specific blocker dofetilide (Tikosyn), clinically known as an antiarrhythmic drug, decreased PAH-associated remodeling, by increasing the arterial lumen opening and by decreasing the arterial wall thickness. Taken together, these findings identify Kv11.1 channels as important players in the function of healthy and pulmonary hypertension-affected lungs in humans and rats. Therefore, Kv11.1 channels should be considered as novel drug targets for the treatment of pulmonary hypertension. Moreover, the Kv11.1 channel blocker dofetilide may have potential in PAH treatment.

\section{Materials and Methods}

\section{Human Tissues}

Samples of $10 \%$ formalin-fixed and paraffin-embedded lung tissues from healthy control subjects and patients with COPD were purchased from National Disease Research Interchange (Philadelphia, PA). The human tissues were selected from donors of both sexes within a postmortem interval of 24 hours. Donors of healthy control tissue had no lung diseases or heart diseases.

\section{Animal Treatment}

Male Sprague-Dawley and Fischer rats were purchased from Charles River Laboratories, Inc. (Wilmington, MA). For the induction of $\mathrm{PAH}$, rats were subcutaneously injected with SU5416 $20 \mathrm{mg} / \mathrm{kg}$ of body weight (MedChemExpress, Monmouth Junction, NJ) and maintained in hypoxia. ${ }^{28-30}$ For hypoxia, animals were placed in a chamber (30 inches wide $\times 20$ inches deep $\times 20$ inches high) regulated by an OxyCycler Oxygen Profile Controller (model A84XOV;
BioSpherix, Redfield, NY) set to maintain $10 \% \mathrm{O}_{2}$ with an influx of $\mathrm{N}_{2}$ gas, located in the animal care facility at the Georgetown University Medical Center (Washington, DC). ${ }^{28,29}$ Ventilation to the outside of the chamber was adjusted to remove $\mathrm{CO}_{2}$, such that its level did not exceed $5000 \mathrm{ppm}$. Animals were fed normal rat chow.

To determine the effects of a Kv.11.1 inhibitor (dofetilide), Sprague-Dawley rats were divided into four groups that were treated with i) dimethyl sulfoxide (DMSO), ii) dofetilide, iii) DMSO + SU5416/hypoxia, and iv) dofetilide + SU5416/hypoxia ( $n=4$ per group). Dofetilide (10 $\mathrm{mg} / \mathrm{kg}$ of body weight), dissolved in DMSO, was injected i.p. before the injection of SU5416. The Georgetown University Animal Care and Use Committee approved the protocols of all of the animal experiments, and the investigation conformed to the NIH's Guide for the Care and Use of Laboratory Animals. ${ }^{31}$

\section{Histologic Measurements}

For histologic examination, lung tissues from both the left and right lungs, all lobes in rats and indicated lobes in humans, were fixed in $10 \%$ formalin and embedded in paraffin. Paraffin-embedded tissues were cut and mounted on glass slides. Tissue sections were subjected to hematoxylin and eosin (H\&E) staining and immunohistochemistry (IHC) analysis using the $\alpha$-smooth muscle actin primary antibody (1:300; catalog number ab5694; Abcam, Cambridge, UK) or Kv11.1 primary antibody (1:1000; catalog number APC-016; Alomone Laboratories, Jerusalem, Israel), followed by a secondary horseradish peroxidase-conjugated antibody (catalog number K4003; Agilent, Santa Clara, CA). Negative-control experiments using Kv11.1 staining were also performed. In these experiments, consecutive sections (where possible) or sections from a region of the tissue that was the same as those in the corresponding noncontrol experiments were stained in the absence of the primary antibody with only the secondary antibody, were stained. No Kv11.1 channel staining was detected in the negative-control experiments.

\section{IHC Image Analysis}

Kv11.1 antibody staining on IHC images was quantified using ImageJ software version 1.52n (NIH, Bethesda, MD; https://imagej.nih.gov/ij). ${ }^{32}$ For each PA analyzed, background-subtracted antibody staining intensity values, in arbitrary units (au), of five different regions were averaged and plotted as a point on scatterplots, with $n$ depicting the number of different PAs analyzed from different humans or rats. The means of the data on the scatterplots are presented as red lines.

For PA wall thickness, 30 vessels per animal were analyzed in each group. Four values of external and internal diameters were measured and averaged for each vessel. The percentage 

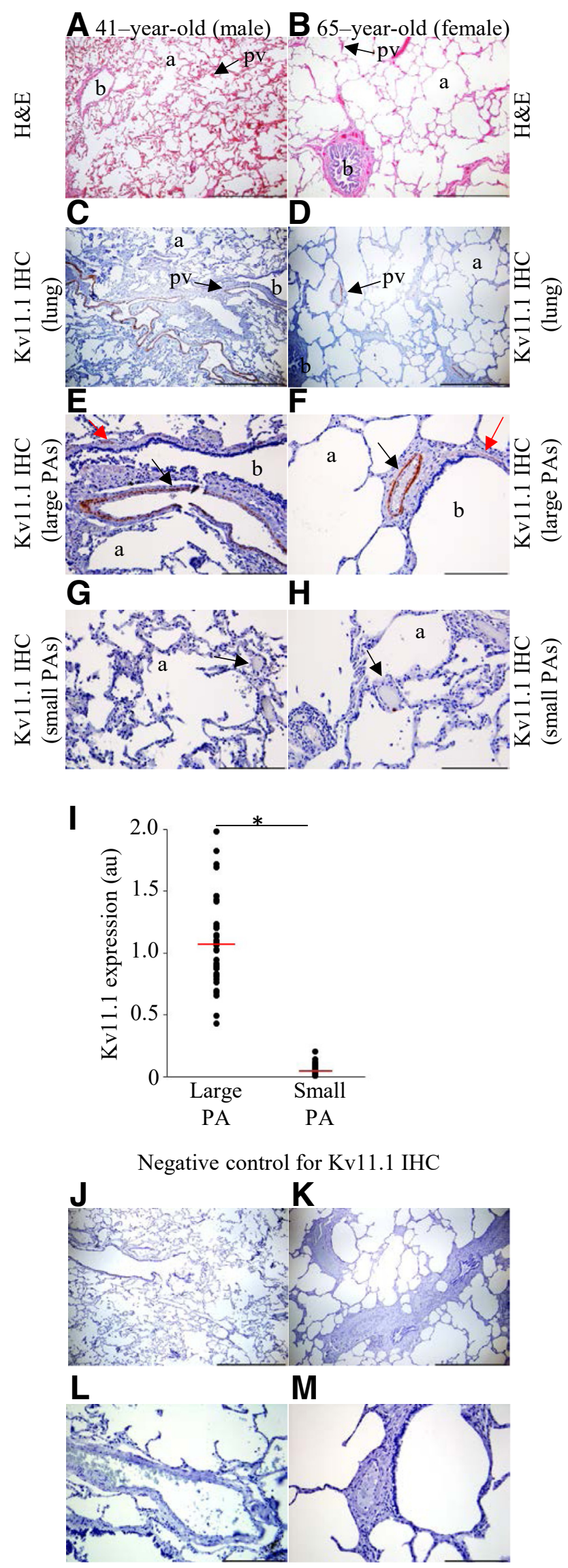

of wall thickness values, calculated as wall thickness divided by vessel radius, were determined using ImageJ.

\section{Statistical Analysis}

Means \pm SEM were calculated. Comparisons between two groups were performed using the $t$-test, whereas comparisons between three or more groups were performed using analysis of variance. $P<0.05$ was considered statistically significant.

\section{Results}

Kv11.1 Channel Expression in the SMCs of Large PAs in the Lungs of Healthy Humans

To investigate Kv11.1 channel expression in human lungs, histologic examination of $\mathrm{H} \& \mathrm{E}-\mathrm{stained}$ postmortem lung tissues from healthy individuals was performed (Figure 1, A and $\mathrm{B}$ ). The examination revealed normal lung structure, with bronchiole, alveoli, and a thin layer of connective tissue between the alveoli and a multitude of pulmonary vessels (Figure 1, A and B). IHC analysis with Kv11.1 channel antibody revealed a robust expression of Kv11.1 channels in the smooth muscle cell (SMC) layer of bronchiole and large-diameter PAs in the lungs of the individuals (Figure 1, C and D). Kv11.1 expression was detected in the large PAs (Figure 1, E and F), and no expression of Kv11.1 channels was detected in the small PAs (Figure 1, G and H). On quantitative comparison of Kv11.1 channel expression in the small and large PAs (Figure 1I), the absence of Kv11.1 channel expression in small PAs was statistically significant. No Kv11.1 channel staining was detected in the negativecontrol experiments (Figure 1, J-M). No statistically significant sex-dependent differences were observed.

Kv11.1 Channel Expression in the SMCs of Small PAs in the Lungs of Humans with COPD

To determine whether the Kv11.1 channel expression level is altered in respiratory disease, channel expression was

Figure 1 Kv11.1 channels are expressed in human lungs. Normal human lung hematoxylin and eosin (H\&E) staining in a 41-year-old man (A) and 65year-old woman (B). Shown are sections of the lung composed of terminal bronchiole (b) and thin-walled alveoli (a). Also shown is a thin layer of alveoli connective tissue and a multitude of pulmonary vessels (pv; arrows). $\mathbf{C}$ and $\mathbf{D}$ : Kv11.1 antibody staining of normal human lung in the 41-year-old man (C, right upper lobe) and 65-year-old woman (D, left lower lobe), with the structural elements denoted in the same manner as in A and B. E and F: Kv11.1 staining in bronchial smooth muscle layer (red arrows) and in the media of pulmonary arteries (PAs) of diameter $>100 \mu \mathrm{m}$ (black arrows) in the 41-yearold man (E) and 65-year-old woman (F). $\mathbf{G}$ and $\mathbf{H}$ : Arrows indicate the absence of Kv11.1 staining in small PAs (diameter $<100 \mu \mathrm{m}$ ). I: Quantification of Kv11.1 channel expression [in arbitrary units (au)] in large and small PAs. J-M: Negative controls for $\mathbf{E}-\mathbf{H}$, correspondingly, with the primary antibody omitted and only the secondary antibody used. The mean of the data is indicated (red lines). $n=37$ per group. ${ }^{*} P<0.05$. Scale bars: $1 \mathrm{~mm}(\mathbf{A}-\mathbf{D})$; $200 \mu \mathrm{m}(\mathbf{E}-\mathbf{H}, \mathbf{J}-\mathbf{M})$. Original magnification: $\times 16(\mathbf{A}-\mathbf{D}) ; \times 200(\mathbf{E}-\mathbf{H}) . \mathrm{IHC}$, immunohistochemistry. 

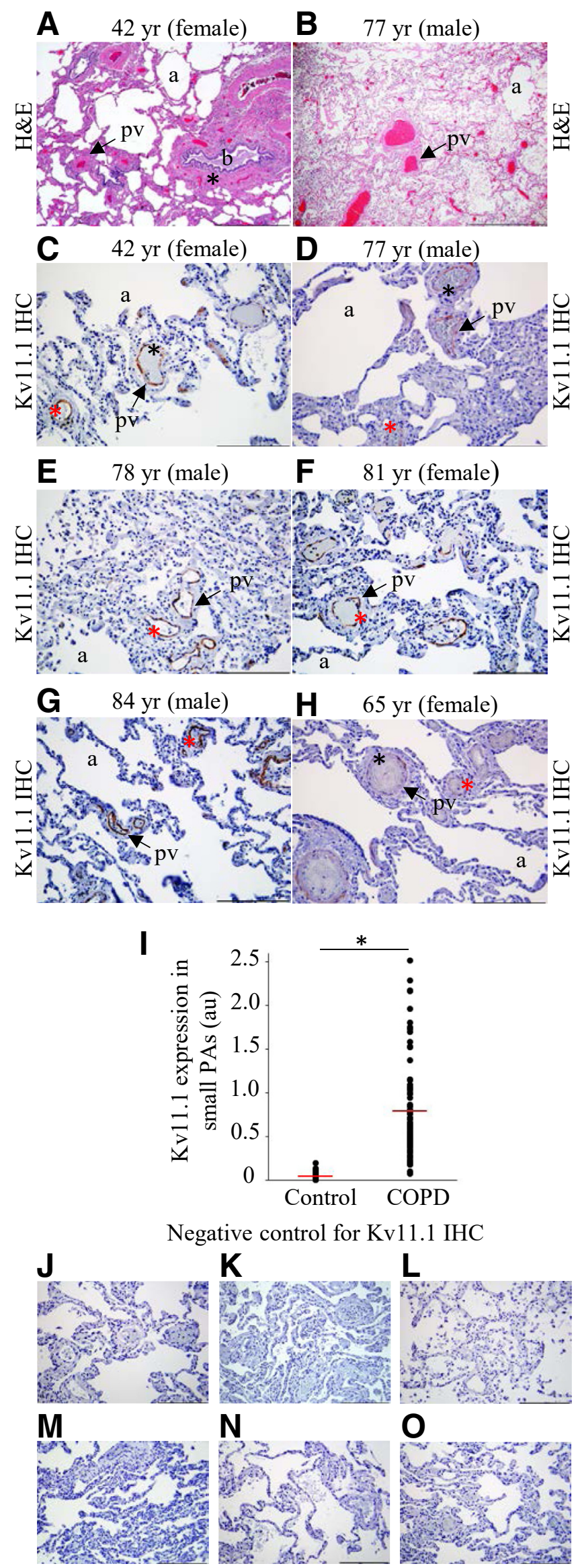

examined in postmortem lung tissue samples from humans with COPD-associated pulmonary hypertension. H\&E staining revealed emphysematous distention and collapse of alveoli, mild edema of the arterial wall, peribronchial and perivascular fibrosis, and thickening of walls of PAs (Figure 2, A and B). On IHC analysis of COPD-affected lungs from individuals of different ages using Kv11.1 channel antibody, Kv11.1 channels were expressed in the media of not only large PAs but also small PAs (Figure 2, $\mathrm{C}-\mathrm{H})$. On quantitative comparison of Kv11.1 channel expression in the small PAs of lungs from healthy humans and COPD patients (Figure 2I), the increase in the expression of Kv11.1 channels in small PAs in COPD patients was statistically significant. No Kv11.1 channel staining was detected in the negative-control experiments (Figure 2, $\mathrm{J}-\mathrm{O}$ ). No statistically significant sex-dependent differences were observed.

Kv11.1 Channel Expression in the SMCs of Large PAs in the Lungs of Healthy Rats

To determine whether the Kv11.1 channel expression observed in healthy human lungs is also present in rat lungs, histologic examination of H\&E-stained lung tissue from healthy Sprague-Dawley rats was conducted. The examination revealed a lung parenchyma with a bronchiole, alveoli, and different-diameter pulmonary vessels of expected sizes (Figure 3A). IHC analysis with the $\alpha$-smooth muscle actin antibody indicated a typical SMC layer of a bronchiole and large PAs and an absence of smooth muscle expression in the small PAs (Figure 3B). The expression of Kv11.1 channels in healthy rat lung tissue was then examined using Kv11.1 channel antibody. IHC analysis revealed robust Kv11.1 expression in the SMC layer of the bronchioles and largediameter PAs (Figure. 3C). No Kv11.1 channel expression was detected in the small PAs (Figure 3C), similar to the observed absence of Kv11.1 channel expression in small PAs in human lungs. On quantitative comparison of Kv11.1 channel expression in the small and large PAs (Figure 3D), the absence of Kv11.1 channel expression in the small PAs was

Figure 2 Kv11.1 channel expression is increased in chronic obstructive pulmonary disease (COPD)-affected human lungs. A and B: Hematoxylin and eosin (H\&E) staining of the lung tissue in individuals of the indicated age and sex showing alveoli emphysema (a), bronchiole (b), peribronchial fibrosis (asterisk), perivascular fibrosis, and pulmonary artery (PA) wall thickness (arrows). C-H: Kv11.1 antibody staining of media of large PAs (diameter $>100 \mu \mathrm{m}$; black asterisks) and small PAs (diameter $<100 \mu \mathrm{m}$; red asterisks) in six different individuals with COPD of the indicated age and sex. Tissue sections in $\mathbf{C}$ and $\mathbf{E}$ are from the right lower lobe; $\mathbf{D}$, from the right upper lobe; $\mathbf{F}$ and $\mathbf{G}$, from the left upper lobe; and $\mathbf{H}$, from the left lower lobe of lungs. Arrows, pulmonary vessels; a, alveoli. I: Quantification of Kv11.1 channel expression (in arbitrary units; au) in small PAs of healthy and COPD-affected lungs. J-0: Negative controls for $\mathbf{C}-\mathbf{H}$, correspondingly, with the primary antibody omitted and only the secondary antibody used. The mean of the data is indicated (red lines). $n=37$ (control); $n=79$ (COPD). ${ }^{*} P<0.05$. Scale bars: $1 \mathrm{~mm}(\mathbf{A}$ and $\mathbf{B}) ; 200 \mu \mathrm{m}(\mathbf{C}-\mathbf{H}, \mathbf{J}-\mathbf{0})$. Original magnification: $\times 16$ (A and B); $\times 200(\mathbf{C}-\mathbf{H})$. IHC, immunohistochemistry. 

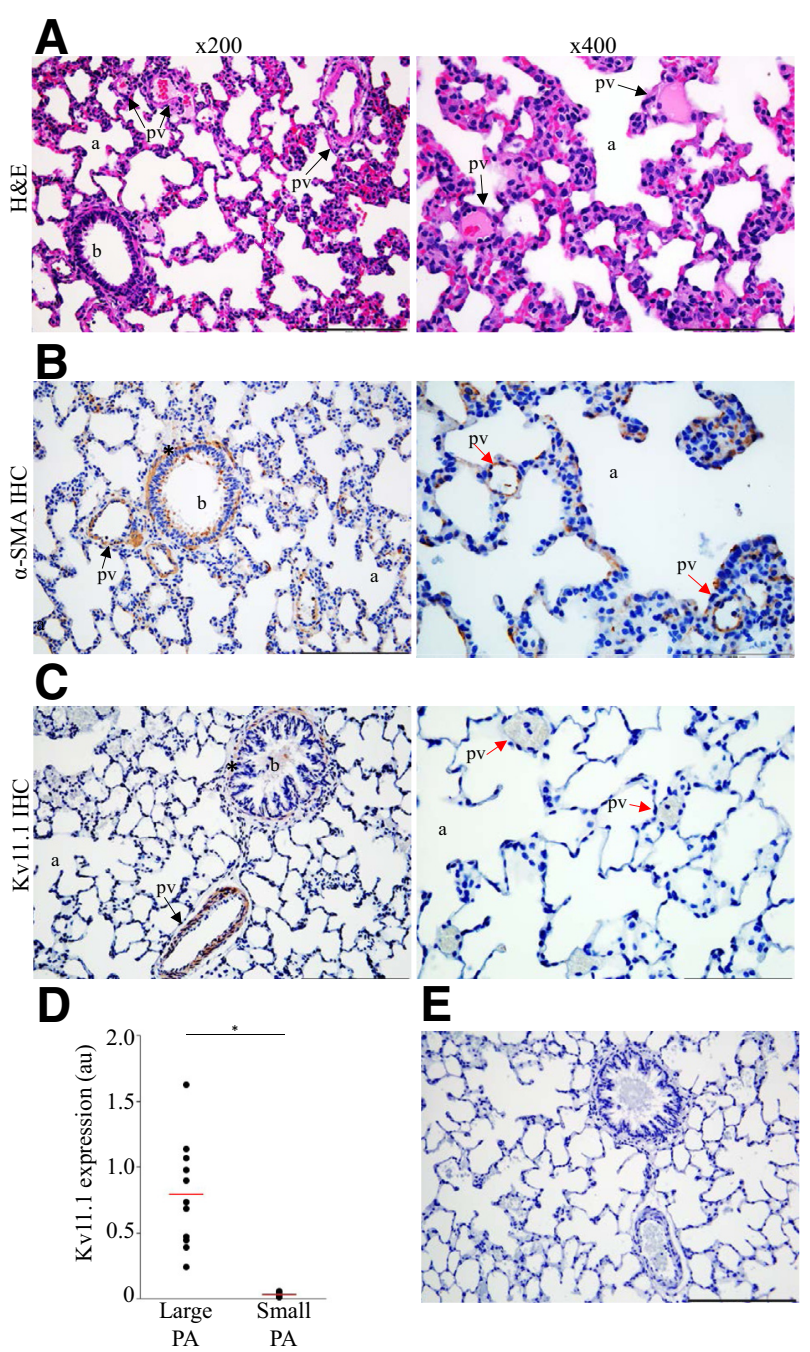

Figure 3 Kv11.1 channels are expressed in healthy rat lungs. A: Hematoxylin and eosin (H\&E) staining of Sprague-Dawley rat lung tissue showing a normal structure of a bronchiole (b), alveoli (a), thin interstitial alveolar wall, and normal pulmonary arteries wall thickness (pv; arrows) in control rats. B: $\alpha$-Smooth muscle actin antibody staining in the smooth muscle cell layer of a bronchiole (asterisk) and in the media of pulmonary arteries (PAs) at diameter $>100 \mu \mathrm{m}$ (black arrow). Absence of $\alpha$-smooth muscle actin antibody staining in the media of PAs at diameter $<100 \mathrm{~mm}$ (red arrows). C: Kv11.1 antibody staining in bronchial SMC layer (asterisk) and in the media of PAs at diameter $>100 \mu \mathrm{m}$ (black arrow). Images in $\times 400$ magnification illustrate the absence of Kv11.1 antibody staining in small PAs (red arrows). D: Quantification of Kv11.1 channel expression in large and small PAs. E: Negative control for $\mathbf{C}$ with the primary antibody omitted and only the secondary antibody used. The mean of the data is indicated (red lines). $n=11$ per group. ${ }^{*} P<0.05$. Scale bars: $100 \mu \mathrm{m}$ (A-C, right panels); 200 $\mu \mathrm{m}(A-C$, left panels, E). Original magnification: $\times 200$ (A-C, left panels); and $\times 400$ (A-C, right panels). IHC, immunohistochemistry.

statistically significant. No Kv11.1 channel staining was detected in the negative-control experiments (Figure 3E).

Kv11.1 Channel Expression in the SMCs of Both Large and Small PAs in Rats with PAH

Next, it was determined whether, similar to humans with COPD, Kv11.1 channel expression is altered in PAH rats.
To induce PAH, Sprague-Dawley rats were injected with SU5416, placed in hypoxia for 3 weeks, and then maintained in normoxia for 5 weeks. ${ }^{28-30}$ The animals were then euthanized and histologic data were obtained. Histologic examination of the H\&E-stained lung tissues revealed the alterations in the lung structure with $\mathrm{PAH}$, including medial hypertrophy and alveolar wall thickening (Figure 4A). These changes lead to the narrowing or occlusion of the lumen in PAs. IHC analysis with $\alpha$-SMA antibody indicated proliferation of SMCs and muscularization of the smalldiameter PAs (Figure 4B) not seen in the normal lung tissue (Figure 3B). IHC analysis with Kv11.1 antibody revealed Kv11.1 channel expression in the SMCs of both the large and small PAs (Figure 4C). The appearance of Kv11.1 channels in small-diameter PAs in rats with PAH was similar to that observed in human lungs with COPD (Figure 2). On quantitative comparison of the Kv11.1 channel expression in the small PAs of lungs from healthy and PAH rats (Figure 4D), the increase in the expression of Kv11.1 channels in PAH rats was statistically significant. No Kv11.1 channel staining was detected in the negativecontrol experiments (Figure 4E).

Changes in Kv11.1 channel expression with PAHassociated vascular remodeling were also observed in Fischer rats at 1,2,3, 4, and 5 weeks after the initiation of SU5416/hypoxia treatment. The time-course experiments revealed that the appearance of the Kv11.1 channel expression in the small-diameter PAs closely followed the time-course of vascular remodeling in PAH and the associated increased muscularization (Figure 5A). No Kv11.1 channel staining was detected in the negative-control experiments (Figure 5B). Quantitative analysis indicated that the changes in the Kv11.1 channel expression with time were statistically significant (Figure 5C).

\section{Effects of Kv11.1 Channel Blocker Dofetilide on PA Wall Remodeling in PAH Rats}

To determine whether pharmacologic inhibition of Kv11.1 channels would affect PAH progression, healthy and PAH rats were treated with the Kv11.1 channel-specific blocker dofetilide.

Lung tissues were examined with H\&E staining. The rats in the control (no PAH) groups (DMSO and dofetilide) had similar lung structure, with no statistical differences in the small and large PA media wall thickness. The PAH rats in the DMSO + SU5416/hypoxia group had increased intimal endothelial cell proliferation, increased small PA wall thickness, and reduced lumen diameter compared to those in the DMSO and dofetilide groups (Figure 6, A and B). Surprisingly, PAH rats in the dofetilide + SU5416/hypoxia group had reduced wall thickness and increased lumen diameter compared with PAH rats in the DMSO + SU5416/ hypoxia group (Figure 6, C and D). Moreover, dofetilide was associated with an elimination of PAH-related occlusion of the lumen. 

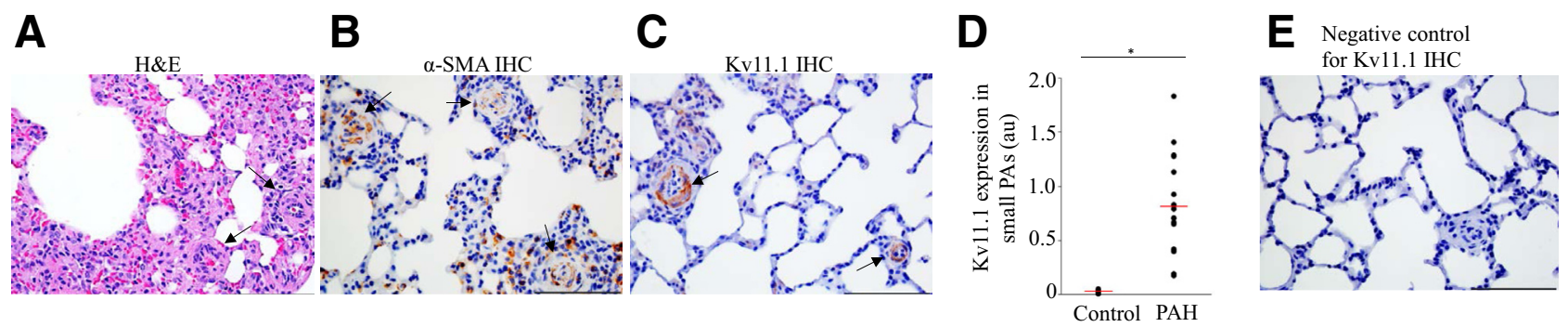

Figure 4 Kv11.1 channel expression increases in pulmonary arterial hypertension (PAH) rats. A: Hematoxylin and eosin (H\&E) staining of lung tissue from Sprague-Dawley rats with PAH shows pulmonary arteries (PAs) wall thickness (arrows). After SU5416 injection, rats were kept in hypoxia for 3 weeks and then maintained in normoxia for 5 weeks. B: $\alpha$-Smooth muscle actin (SMA) antibody staining in the media of small PAs (brown; arrows). C: Kv11.1 antibody staining in the media of small PAs (brown; arrows). D: Quantification of Kv11.1 channel expression (in arbitrary units; au) in small PAs of healthy and PAH rat lungs. E: Negative control for $\mathbf{C}$ with the primary antibody omitted and only the secondary antibody used. The mean of the data is indicated (red lines). $n=11$ (control); $n=18(\mathrm{PAH}) .{ }^{*} P<0.05$. Scale bars $=100 \mu \mathrm{m}(\mathbf{A}-\mathbf{C}$ and $\mathbf{E})$. Original magnification, $\times 400(\mathbf{A}-\mathbf{C}$ and $\mathbf{E})$. IHC, immunohistochemistry.

\section{Discussion}

The findings from this study demonstrate that Kv11.1 channels are expressed in the lungs of rats and humans. In healthy lung tissues, Kv11.1 expression was limited to the SMC layer of large PAs (diameter $\geq 100 \mu \mathrm{m}$ ). In rats with PAH and humans with COPD-associated pulmonary hypertension, Kv11.1 channel expression was detected not only in the large PAs but also in the small PAs (diameter $<100 \mu \mathrm{m}$ ). Importantly, treatment with the Kv11.1 channel blocker dofetilide was associated with reduced vascular remodeling in PAH rats and maintenance of media wall thickness at the level seen in healthy rats. Dofetilide is clinically used to treat atrial fibrillations. These results provide a rationale for further exploring the modulation of Kv11.1 channels as a potential treatment of pulmonary hypertension and repurposing of dofetilide for the treatment of PAH and COPD patients.

Kv11.1 channels are abundantly expressed in the heart ${ }^{33,34}$ and brain. ${ }^{11,34}$ In the heart, Kv11.1 channels are expressed in cardiac myocytes, ${ }^{35,36}$ and their function is to repolarize cardiac action potential. ${ }^{1-4}$ In the brain, Kv11.1 channels are expressed in various regions, and their function is to regulate neuronal excitability. ${ }^{11,37-39}$ In addition to the heart and brain, Kv11.1 channels are also expressed in a variety of other tissues and organs, ${ }^{10}$ including retina ${ }^{13}$; chromaffin cells ${ }^{40}$; and SMCs of gallbladder, ${ }^{14}$ stomach, ${ }^{15}$ and intestines. ${ }^{16-18}$ Although Kv11.1 channel mRNA was detected in rat lung tissue, ${ }^{34}$ the pattern of Kv11.1 channel protein expression has not been known. Histologic examination of human and rat lung tissues indicated that Kv11.1 channels were expressed in the SMC layer of large-diameter PAs (Figures 1, E and F, and 3C). Interestingly, in healthy lungs, Kv11.1 channel expression was not detected in the SMC layer of small-diameter PAs (Figures 1, G and $\mathrm{H}$, and $3 \mathrm{C}$ ). These findings indicate that Kv11.1 channels are part of the ion channel landscape of the lung smooth muscles and should be considered a novel target for their modulation.

Importantly, in humans with COPD and in rats with PAH, the expression of Kv11.1 channels was increased and was detected in not only large but also small PAs (Figures 2 and 4). The increased Kv11.1 expression in pulmonary hypertension suggests that Kv11.1 channel expression is linked to vascular remodeling. This idea is further supported by the time-course of changes in Kv11.1 channel expression. The Kv11.1 channel expression increased gradually in the PAH rats, with the time-course closely following that of the arterial wall thickening (Figure 5).

To test whether pharmacologic inhibition of Kv11.1 channels could affect vascular remodeling in PAH, the Kv11.1 channel blocker dofetilide was injected at the time of PAH induction. Dofetilide was associated with substantial inhibition of PAH-associated vascular remodeling in the dofetilide-treated rats (Figure 6). Dofetilide was also associated with increased lumen diameter and decreased PA wall thickness, to the levels seen in the control rats without PAH. These findings suggest that inhibition of Kv11.1 channels has a protective effect in pulmonary hypertension. The effect of dofetilide is opposite to the expected role based on the conventional role of $\mathrm{K}^{+}$channels as hyperpolarization generators in SMCs. Kv11.1 channels have been shown to regulate the excitability of SMCs in various tissues, including epididymal duct, ${ }^{41}$ gastrointestinal tract, ${ }^{16,18,42}$ gallbladder, ${ }^{14}$ and portal vein, ${ }^{43}$ by contributing to the resting membrane potential and to the repolarization phase of the action potential. ${ }^{36,44,45}$ Based on those previous reports, Kv11.1 channels would be expected to hyperpolarize SMCs in the lung tissue and decrease the SMC contractile tone. Therefore, Kv11.1 channels are expected to play a protective role in PAH, and Kv11.1 channel blockers would then exacerbate PAH-induced vascular remodeling, which is opposite to what we report here.

These findings may be reconciled based on another wellestablished role of Kv11.1 channels: the regulation of cell proliferation during normal development and cancer. ${ }^{46}$ Kv11.1 channel-mediated hyperpolarization is crucial for the progression of the normal cell cycle. ${ }^{47,48}$ Importantly, the expression of Kv11.1 channels is up-regulated in many cancerous tissues, ${ }^{46,49}$ and the inhibition of Kv11.1 channel activity with Kv11.1 channel-specific blockers decreases 

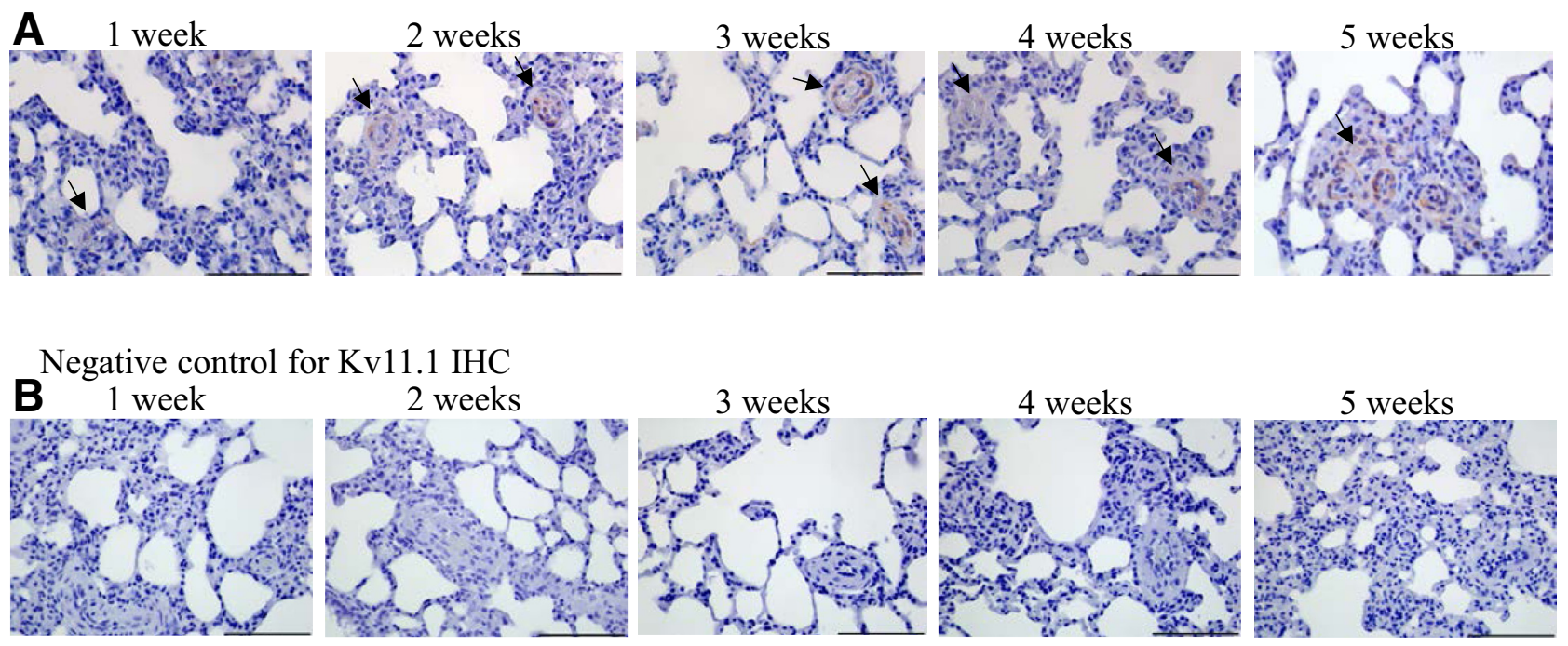

C

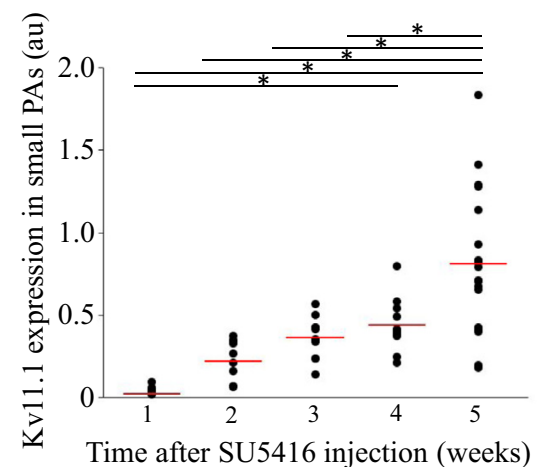

Figure 5 Kv11.1 channel expression gradually increases with the development of pulmonary arterial hypertension (PAH) in rats. A: Time course examination of Kv11.1 expression in PAH rats (brown; arrows). For this experiment Fischer rats were injected with SU5416, placed in hypoxia for 3 weeks, and then maintained in normoxia for 2 weeks to obtain time points of $0,1,2,3,4$, and 5 weeks after the SU5416 injection. B: Negative control for the corresponding images in $\mathbf{A}$ with the primary antibody omitted and only the secondary antibody used. C: Quantification of the changes in Kv11.1 channel expression [in arbitrary units (au)] with PAH development in small pulmonary arteries (PAs). The mean of the data is indicated (red lines). $n=10 .{ }^{*} P<0.05$. Scale bars $=100 \mu \mathrm{m}(\mathbf{A}$ and $\mathbf{B})$. Original magnification, $\times 400$ (A). IHC, immunohistochemistry.

cell proliferation. ${ }^{50}$ Although the mechanism of Kv11.1 channel regulation of tumor progression is not completely clear, a few potential leads are worth mentioning. The promoter region of Kv11.1 channel gene contains binding sites for many oncoproteins, enabling Kv11.1 channels to regulate cell-growth in response to the activation of oncogenes. ${ }^{51}$ There is evidence of an interaction between Kv11.1 channels and $\beta_{1}$-integrins that in turn affects the migration. ${ }^{52}$ We hypothesize that, similar to that in cancer, the growth of SMCs in PAH is associated with overexpression of Kv11.1 channels, and that the application of the Kv11.1 channel blocker dofetilide decreases SMC growth and PAHassociated vascular remodeling.

Dofetilide is a drug approved by the US Food and Drug Administration for use in the treatment of irregular heartbeat. As other Class III antiarrhythmic drugs, it acts by prolonging the cardiac action potential via blocking Kv11.1 channels in the heart. ${ }^{53}$ In cases of cardiac arrhythmia associated with faster-than-normal heart rate, such as tachycardia, delaying cardiac repolarization and reducing
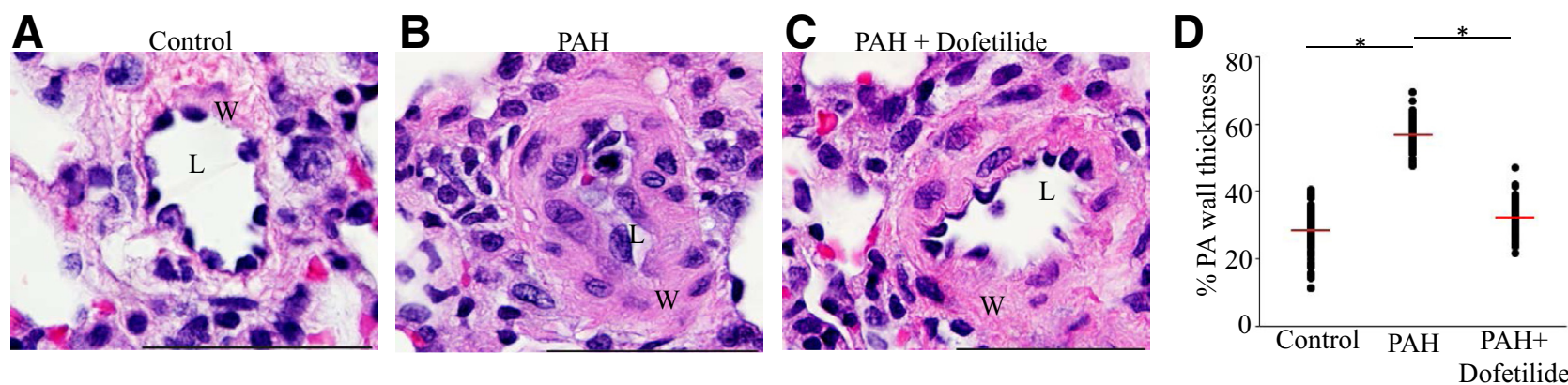

Figure 6 Dofetilide inhibits pulmonary vascular remodeling. Representative images of pulmonary vessels of control (A), pulmonary arterial hypertension (PAH) (B), and PAH and dofetilide treated (C) Sprague-Dawley rats (hematoxylin and eosin stain). D: Percent PA wall thickness for the three indicated groups. Data are expressed as means (red lines) \pm SEM. ${ }^{*} P<0.05$. Scale bars $=50 \mu \mathrm{m}(\mathbf{A}-\mathbf{C})$. Original magnification, $\times 1000(\mathbf{A}-\mathbf{C}) . \mathrm{L}$, pulmonary artery lumen; W, pulmonary artery wall. 
the heart rate by blocking Kv11.1 channels would then stabilize the heart rate. ${ }^{54,55}$ The findings from this study indicate that Kv11.1 channels are expressed in the lungs and their inhibition by dofetilide prevents the symptoms of arterial muscularization associated with PAH. These findings warrant further investigation of the potentially positive effects of dofetilide in patients with PAH.

In summary, in healthy humans and rats, Kv11.1 channels are expressed in the SMCs of large PAs only, whereas in humans with COPD and rats with PAH, Kv11.1 channels are expressed in both the large and small PAs. Inhibition of Kv11.1 channels by dofetilide was associated with decreased PAH-associated vascular remodeling, suggesting that Kv11.1 channel blockers might have potential in PAH treatment.

\section{References}

1. Schonherr R, Heinemann SH: Molecular determinants for activation and inactivation of HERG, a human inward rectifier potassium channel. J Physiol 1996, 493:635-642

2. Smith PL, Baukrowitz T, Yellen G: The inward rectification mechanism of the HERG cardiac potassium channel. Nature 1996, 379: 833-836

3. Spector PS, Curran ME, Keating MT, Sanguinetti MC: Class III antiarrhythmic drugs block HERG, a human cardiac delayed rectifier $\mathrm{K}+$ channel. Open-channel block by methanesulfonanilides. Circ Res 1996, 78:499-503

4. Trudeau MC, Warmke JW, Ganetzky B, Robertson GA: HERG, a human inward rectifier in the voltage-gated potassium channel family. Science 1995, 269:92-95

5. Curran ME, Splawski I, Timothy KW, Vincent GM, Green ED, Keating MT: A molecular basis for cardiac arrhythmia: HERG mutations cause long QT syndrome. Cell 1995, 80:795-803

6. Kiehn J, Lacerda AE, Wible B, Brown AM: Molecular physiology and pharmacology of HERG. Single-channel currents and block by dofetilide. Circulation 1996, 94:2572-2579

7. Sanguinetti MC, Jurkiewicz NK, Scott A, Siegl PK: Isoproterenol antagonizes prolongation of refractory period by the class III antiarrhythmic agent E-4031 in guinea pig myocytes. Mechanism of action. Circ Res 1991, 68:77-84

8. Zhou Z, Gong Q, Epstein ML, January CT: HERG channel dysfunction in human long QT syndrome. Intracellular transport and functional defects. J Biol Chem 1998, 273:21061-21066

9. Bianchi L, Wible B, Arcangeli A, Taglialatela M, Morra F, Castaldo P, Crociani O, Rosati B, Faravelli L, Olivotto M, Wanke E: hERG encodes a $\mathrm{K}+$ current highly conserved in tumors of different histogenesis: a selective advantage for cancer cells? Cancer Res 1998, $58: 815-822$

10. Babcock JJ, Li M: hERG channel function: beyond long QT. Acta Pharmacol Sin 2013, 34:329-335

11. Guasti L, Cilia E, Crociani O, Hofmann G, Polvani S, Becchetti A, Wanke E, Tempia F, Arcangeli A: Expression pattern of the ether-ago-go-related (ERG) family proteins in the adult mouse central nervous system: evidence for coassembly of different subunits. J Comp Neurol 2005, 491:157-174

12. Papa M, Boscia F, Canitano A, Castaldo P, Sellitti S, Annunziato L, Taglialatela M: Expression pattern of the ether-a-gogo-related (ERG) $\mathrm{K}+$ channel-encoding genes ERG1, ERG2, and ERG3 in the adult rat central nervous system. J Comp Neurol 2003, 466:119-135

13. Cordeiro S, Guseva D, Wulfsen I, Bauer CK: Expression pattern of Kv11 (ether a-go-go-related gene; erg) $\mathrm{K}+$ channels in the mouse retina. PLoS One 2011, 6:e29490
14. Parr E, Pozo MJ, Horowitz B, Nelson MT, Mawe GM: ERG K+ channels modulate the electrical and contractile activities of gallbladder smooth muscle. Am J Physiol Gastrointest Liver Physiol 2003, 284:G392-G398

15. Ohya S, Asakura K, Muraki K, Watanabe M, Imaizumi Y: Molecular and functional characterization of ERG, KCNQ, and KCNE subtypes in rat stomach smooth muscle. Am J Physiol Gastrointest Liver Physiol 2002, 282:G277-G287

16. Farrelly AM, Ro S, Callaghan BP, Khoyi MA, Fleming N, Horowitz B, Sanders KM, Keef KD: Expression and function of KCNH2 (HERG) in the human jejunum. Am J Physiol Gastrointest Liver Physiol 2003, 284:G883-G895

17. Lamarca V, Grasa L, Fagundes DS, Arruebo MP, Plaza MA, Murillo MD: $\mathrm{K}+$ channels involved in contractility of rabbit small intestine. J Physiol Biochem 2006, 62:227-236

18. Lillich JD, Rakestraw PC, Roussel AJ, Finley MR, Ganta S, Freeman LC: Expression of the ether-a-go-go (ERG) potassium channel in smooth muscle of the equine gastrointestinal tract and influence on activity of jejunal smooth muscle. Am J Vet Res 2003, $64: 267-272$

19. Bartoszewski R, Matalon S, Collawn JF: Ion channels of the lung and their role in disease pathogenesis. Am J Physiol Lung Cell Mol Physiol 2017, 313:L859-L872

20. Shujaat A, Minkin R, Eden E: Pulmonary hypertension and chronic cor pulmonale in COPD. Int J Chron Obstruct Pulmon Dis 2007, 2: 273-282

21. Boucherat O, Chabot S, Antigny F, Perros F, Provencher S, Bonnet S: Potassium channels in pulmonary arterial hypertension. Eur Respir J 2015, 46:1167-1177

22. Austin ED, Loyd JE: The genetics of pulmonary arterial hypertension. Circ Res 2014, 115:189-202

23. Dodson MW, Brown LM, Elliott CG: Pulmonary arterial hypertension. Heart Fail Clin 2018, 14:255-269

24. Farber HW, Loscalzo J: Pulmonary arterial hypertension. N Engl J Med 2004, 351:1655-1665

25. Higenbottam T: Pulmonary hypertension and chronic obstructive pulmonary disease: a case for treatment. Proc Am Thorac Soc 2005, 2:12-19

26. Weir EK, Olschewski A: Role of ion channels in acute and chronic responses of the pulmonary vasculature to hypoxia. Cardiovasc Res 2006, 71:630-641

27. Dunham-Snary KJ, Wu D, Sykes EA, Thakrar A, Parlow LRG, Mewburn JD, Parlow JL, Archer SL: Hypoxic pulmonary vasoconstriction: from molecular mechanisms to medicine. Chest 2017, 151: $181-192$

28. Ibrahim YF, Shults NV, Rybka V, Suzuki YJ: Docetaxel reverses pulmonary vascular remodeling by decreasing autophagy and resolves right ventricular fibrosis. J Pharmacol Exp Ther 2017, 363: $20-34$

29. Ibrahim YF, Wong CM, Pavlickova L, Liu L, Trasar L, Bansal G, Suzuki YJ: Mechanism of the susceptibility of remodeled pulmonary vessels to drug-induced cell killing. J Am Heart Assoc 2014, 3: e000520

30. Oka M, Homma N, Taraseviciene-Stewart L, Morris KG, Kraskauskas D, Burns N, Voelkel NF, McMurtry IF: Rho kinase-mediated vasoconstriction is important in severe occlusive pulmonary arterial hypertension in rats. Circ Res 2007, 100:923-929

31. Committee for the Update of the Guide for the Care and Use of Laboratory AnimalsNational Research Council: Guide for the Care and Use of Laboratory Animals: Eighth Edition. Washington, DC, National Academies Press, 2011

32. Schneider CA, Rasband WS, Eliceiri KW: NIH Image to ImageJ: 25 years of image analysis. Nat Methods 2012, 9:671-675

33. Shi W, Wymore R, Yu H, Wu J, Wymore RT, Pan Z, Robinson RB, Dixon JE, McKinnon D, Cohen IS: Distribution and prevalence of hyperpolarization-activated cation channel ( $\mathrm{HCN})$ mRNA expression in cardiac tissues. Circ Res 1999, 85:e1-e6 
34. Wymore RS, Gintant GA, Wymore RT, Dixon JE, McKinnon D, Cohen IS: Tissue and species distribution of mRNA for the IKr-like K+ channel, erg. Circ Res 1997, 80:261-268

35. Melnyk P, Ehrlich JR, Pourrier M, Villeneuve L, Cha TJ, Nattel S: Comparison of ion channel distribution and expression in cardiomyocytes of canine pulmonary veins versus left atrium. Cardiovasc Res 2005, 65:104-116

36. Sanguinetti MC, Jiang C, Curran ME, Keating MT: A mechanistic link between an inherited and an acquired cardiac arrhythmia: HERG encodes the IKr potassium channel. Cell 1995, 81:299-307

37. Titus SA, Warmke JW, Ganetzky B: The Drosophila erg K+ channel polypeptide is encoded by the seizure locus. J Neurosci 1997, 17: 875-881

38. Sacco T, Bruno A, Wanke E, Tempia F: Functional roles of an ERG current isolated in cerebellar Purkinje neurons. J Neurophysiol 2003, 90:1817-1828

39. Furlan F, Taccola G, Grandolfo M, Guasti L, Arcangeli A, Nistri A, Ballerini L: ERG conductance expression modulates the excitability of ventral horn GABAergic interneurons that control rhythmic oscillations in the developing mouse spinal cord. J Neurosci 2007, 27: 919-928

40. Gullo F, Ales E, Rosati B, Lecchi M, Masi A, Guasti L, CanoAbad MF, Arcangeli A, Lopez MG, Wanke E: ERG K+ channel blockade enhances firing and epinephrine secretion in rat chromaffin cells: the missing link to LQT2-related sudden death? FASEB J 2003, $17: 330-332$

41. Mewe M, Wulfsen I, Schuster AM, Middendorff R, Glassmeier G, Schwarz JR, Bauer CK: Erg K+ channels modulate contractile activity in the bovine epididymal duct. Am J Physiol Regul Integr Comp Physiol 2008, 294:R895-R904

42. Akbarali HI, Thatte H, He XD, Giles WR, Goyal RK: Role of HERGlike $\mathrm{K}(+)$ currents in opossum esophageal circular smooth muscle. Am J Physiol 1999, 277:C1284-C1290

43. Ohya S, Horowitz B, Greenwood IA: Functional and molecular identification of ERG channels in murine portal vein myocytes. Am J Physiol Cell Physiol 2002, 283:C866-C877

44. Zhu Y, Golden CM, Ye J, Wang XY, Akbarali HI, Huizinga JD: ERG $\mathrm{K}+$ currents regulate pacemaker activity in ICC. Am J Physiol Gastrointest Liver Physiol 2003, 285:G1249-G1258
45. Mewe M, Bauer CK, Schwarz JR, Middendorff R: Mechanisms regulating spontaneous contractions in the bovine epididymal duct. Biol Reprod 2006, 75:651-659

46. Vandenberg JI, Perry MD, Perrin MJ, Mann SA, Ke Y, Hill AP: hERG $K(+)$ channels: structure, function, and clinical significance. Physiol Rev 2012, 92:1393-1478

47. Arcangeli A, Bianchi L, Becchetti A, Faravelli L, Coronnello M, Mini E, Olivotto M, Wanke E: A novel inward-rectifying K+ current with a cell-cycle dependence governs the resting potential of mammalian neuroblastoma cells. J Physiol 1995, 489(Pt 2):455-471

48. Crociani O, Guasti L, Balzi M, Becchetti A, Wanke E, Olivotto M, Wymore RS, Arcangeli A: Cell cycle-dependent expression of HERG1 and HERG1B isoforms in tumor cells. J Biol Chem 2003, 278: 2947-2955

49. Jehle J, Schweizer PA, Katus HA, Thomas D: Novel roles for hERG $\mathrm{K}(+)$ channels in cell proliferation and apoptosis. Cell Death Dis 2011, 2:e193

50. Smith GA, Tsui HW, Newell EW, Jiang X, Zhu XP, Tsui FW, Schlichter LC: Functional up-regulation of HERG K+ channels in neoplastic hematopoietic cells. J Biol Chem 2002, 277:18528-18534

51. Lin H, Xiao J, Luo X, Wang H, Gao H, Yang B, Wang Z: Overexpression HERG $\mathrm{K}(+)$ channel gene mediates cell-growth signals on activation of oncoproteins SP1 and NF-kappaB and inactivation of tumor suppressor Nkx3.1. J Cell Physiol 2007, 212:137-147

52. Cherubini A, Hofmann G, Pillozzi S, Guasti L, Crociani O, Cilia E, Di Stefano P, Degani S, Balzi M, Olivotto M, Wanke E, Becchetti A, Defilippi P, Wymore R, Arcangeli A: Human ether-a-go-go-related gene 1 channels are physically linked to beta1 integrins and modulate adhesion-dependent signaling. Mol Biol Cell 2005, 16:2972-2983

53. Ficker E, Jarolimek W, Kiehn J, Baumann A, Brown AM: Molecular determinants of dofetilide block of HERG $\mathrm{K}+$ channels. Circ Res 1998, 82:386-395

54. Clancy CE, Kurokawa J, Tateyama M, Wehrens XH, Kass RS: K+ channel structure-activity relationships and mechanisms of druginduced QT prolongation. Annu Rev Pharmacol Toxicol 2003, 43: $441-461$

55. Sanguinetti MC, Jurkiewicz NK: Two components of cardiac delayed rectifier $\mathrm{K}+$ current. Differential sensitivity to block by class III antiarrhythmic agents. J Gen Physiol 1990, 96:195-215 